\title{
Hardy's Setup and Elements of Reality
}

\author{
Louis Marchildon \\ Département de physique, Université du Québec, \\ Trois-Rivières, Qc. Canada G9A 5H7 \\ email: marchild@uqtr.ca
}

\begin{abstract}
Several arguments have been proposed some years ago, attempting to prove the impossibility of defining Lorentz-invariant elements of reality. Here I revisit that question, and bring a number of additional considerations to it. I will first analyze Hardy's argument, which was meant to show that Lorentz-invariant elements of reality are indeed inconsistent with quantum mechanics. I will then investigate to what extent the light cone associated with an event can be used to define Lorentz-invariant elements of reality. It turns out to be possible, but elements of reality associated with a product of two commuting operators will not always be equal to the product of elements of reality associated with each operator. I will finally examine a number of ways in which the paradoxical features of Hardy's experiment can be better understood.
\end{abstract}

\section{Introduction}

The notion of 'element of reality' was introduced in the famous Einstein, Podolsky and Rosen (EPR) paper [1], as an attribute of a physical quantity whose value can be predicted with certainty without disturbing the system. To avoid the ambiguity of the phrase 'without disturbing the system,' Redhead [2] later gave the following sufficient condition for the existence of an element of reality, hereafter called ER1:

If we can predict with certainty, or at any rate with probability one, the result of measuring a physical quantity at time $t$, then 
at the time $t$ there exists an element of reality corresponding to the physical quantity and having a value equal to the predicted measurement result. [ER1]

Several interpretations of quantum mechanics involving various kinds of elements of reality were proposed after the EPR paper, Bohmian mechanics in particular [3]. Originally developed as nonrelativistic theories, they have been notoriously difficult to reconcile with the special theory of relativity. Eventually, the question was raised whether Lorentz-invariant elements of reality are inconsistent with quantum mechanics [4, 5, 6].

In this paper I will first analyze Hardy's argument, which was meant to show that Lorentz-invariant elements of reality are indeed inconsistent with quantum mechanics. I will then investigate to what extent the light cone associated with an event can be used to define Lorentz-invariant elements of reality. It turns out to be possible, but these elements of reality won't satisfy the so-called product rule, i.e. an element of reality associated with a product of two commuting operators will not always be equal to the product of elements of reality associated with each operator [7, 8]. I will finally examine how, in several interpretations of quantum mechanics, the paradoxical features of Hardy's experiment can be better understood.

\section{Hardy's argument}

Hardy's gedanken experiment [4] is illustrated in Fig. 1. Two Mach-Zehndertype interferometers are set up, one for electrons ( $\mathrm{MZ}^{-}$, lower right) and one for positrons $\left(\mathrm{MZ}^{+}\right.$, upper left). Electron (positron) states are prepared with initial state vectors $\left|s^{-}\right\rangle\left(\left|s^{+}\right\rangle\right)$, which are wave packets concentrated around paths $s^{-}\left(s^{+}\right)$indicated in Fig. 1. There is annihilation with unit probability if the electron and positron wave packets meet at point $\mathrm{P}$.

Beam splitters $\mathrm{BS}^{ \pm}$and $\mathrm{BS}^{ \pm}$act so that

$$
\begin{aligned}
& \left|s^{ \pm}\right\rangle \rightarrow \frac{1}{\sqrt{2}}\left(i\left|u^{ \pm}\right\rangle+\left|v^{ \pm}\right\rangle\right), \\
& \left|u^{ \pm}\right\rangle \rightarrow \frac{1}{\sqrt{2}}\left(\left|c^{ \pm}\right\rangle+i\left|d^{ \pm}\right\rangle\right), \\
& \left|v^{ \pm}\right\rangle \rightarrow \frac{1}{\sqrt{2}}\left(i\left|c^{ \pm}\right\rangle+\left|d^{ \pm}\right\rangle\right) .
\end{aligned}
$$




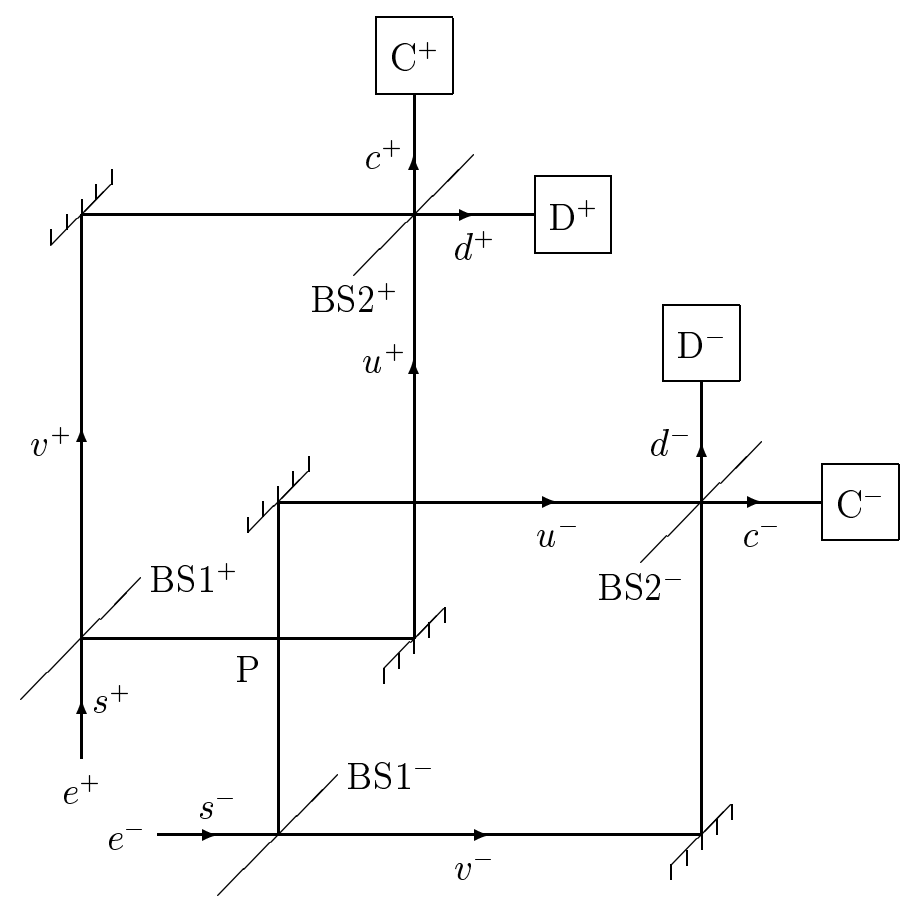

Figure 1: Hardy's thought experiment with two Mach-Zehnder-type interferometers

State vectors $\left|u^{ \pm}\right\rangle,\left|v^{ \pm}\right\rangle,\left|c^{ \pm}\right\rangle$and $\left|d^{ \pm}\right\rangle$are wave packets concentrated around associated paths in Fig. 1.

The evolution of the joint particles' state vector is explained in Ref. [4, from which we quote the following results. Henceforth $|\gamma\rangle$ represents photon states resulting from electron-positron annihilation.

In any run of the experiment, there is a Lorentz frame $F^{-}$in which the electron wave packet has gone through $\mathrm{BS} 2^{-}$before the positron wave packet has reached $\mathrm{BS}^{+}$. During that time, the state vector is given by:

$$
\frac{1}{2 \sqrt{2}}\left(-\sqrt{2}|\gamma\rangle-\left|u^{+}\right\rangle\left|c^{-}\right\rangle+2 i\left|v^{+}\right\rangle\left|c^{-}\right\rangle+i\left|u^{+}\right\rangle\left|d^{-}\right\rangle\right)
$$

Similarly, there is a Lorentz frame $F^{+}$in which the positron wave packet has gone through $\mathrm{BS}^{+}$before the electron wave packet has reached $\mathrm{BS}^{-}$. 
During that time, the state vector is given by:

$$
\frac{1}{2 \sqrt{2}}\left(-\sqrt{2}|\gamma\rangle-\left|c^{+}\right\rangle\left|u^{-}\right\rangle+2 i\left|c^{+}\right\rangle\left|v^{-}\right\rangle+i\left|d^{+}\right\rangle\left|u^{-}\right\rangle\right)
$$

Furthermore, before the positron wave packet has gone through $\mathrm{BS} 2^{+}$and the electron wave packet has gone through $\mathrm{BS}^{-}$, the state vector in all frames is given by:

$$
\frac{1}{2}\left(-|\gamma\rangle+i\left|u^{+}\right\rangle\left|v^{-}\right\rangle+i\left|v^{+}\right\rangle\left|u^{-}\right\rangle+\left|v^{+}\right\rangle\left|v^{-}\right\rangle\right)
$$

Finally, when both the electron and the positron wave packets have gone through the second beam splitters, the state vector in all frames is given by

$$
\begin{gathered}
\frac{1}{4}\left(-2|\gamma\rangle-3\left|c^{+}\right\rangle\left|c^{-}\right\rangle+i\left|c^{+}\right\rangle\left|d^{-}\right\rangle\right. \\
\left.+i\left|d^{+}\right\rangle\left|c^{-}\right\rangle-\left|d^{+}\right\rangle\left|d^{-}\right\rangle\right) .
\end{gathered}
$$

To argue against relativistic elements of reality, Hardy proposes a sufficient condition for their existence and a necessary condition for their Lorentz invariance. The sufficient condition essentially coincides with Redhead's ER1. The necessary condition, hereafter called LI1, simply reads as:

The value of an element of reality corresponding to a Lorentzinvariant observable is itself Lorentz invariant. [LI1]

I shall denote an element of reality associated with an observable $A$ by $f(A)$. Whenever ER1 is satisfied for $A$, then $f(A)$ coincides with an eigenvalue of $A$, a real number.

Suppose that in frame $F^{-}$, an electron is detected in $D^{-}$. From Eq. (4), one can predict with certainty that a measurement of the observable $U^{+}=$ $\left|u^{+}\right\rangle\left\langle u^{+}\right|$will yield the value 1 . Hence $U^{+}$is an element of reality, and $f\left(U^{+}\right)=1$. Likewise suppose that in frame $F^{+}$, a positron is detected in $D^{+}$. From Eq. (5) , this implies that $U^{-}=\left|u^{-}\right\rangle\left\langle u^{-}\right|$is an element of reality, and $f\left(U^{-}\right)=1$. According to Eq. (7), both these situations will occur together, on average, in one of every sixteen runs. In any such case, LI1 implies that both $U^{+}$and $U^{-}$are elements of reality. But Hardy claims that

$$
f\left(U^{+}\right) f\left(U^{-}\right)=1 \Rightarrow f\left(U^{+} U^{-}\right)=1 .
$$


Hence in every run where an electron is detected in $D^{-}$and a positron is detected in $D^{+}$, we obtain that $f\left(U^{+} U^{-}\right)=1$.

From Eq. (6), however, we can predict with certainty that a measurement of the observable $U^{+} U^{-}$will yield the value 0 . Thus $f\left(U^{+} U^{-}\right)=0$, which contradicts the result of the previous paragraph.

The upshot is that there seems to be no way to assign elements of reality in a relativistically invariant way.

\section{Analysis}

I have argued elsewhere 9] that the inference made in (8) assumes that ER1 is not only a sufficient, but also a necessary condition. This conclusion is reinforced by the fact that variants of Hardy's argument [6] assume the validity of the product rule for commuting operators:

$$
f\left(U^{+}\right) f\left(U^{-}\right)=f\left(U^{+} U^{-}\right) .
$$

Indeed one can show [9] that any real-valued function $f$ which (i) is defined on a maximal set of commuting Hermitian operators, (ii) satisfies Eq. (9), and (iii) is 1 on some but not all one-dimensional projectors in the set, singles out a one-dimensional subspace of the state space, i.e. there is a unique one-dimensional projector in the set on which $f=1$. If the element of reality that this function assigns is identified with an eigenvalue of a quantum observable, then a unique state vector is singled out by the specification that $f=1$. Hence it leads to the most definite predictions that quantum mechanics allows.

Since the contradiction obtained in Sec. 2 involves somewhat more than the sufficient condition ER1 and the necessary condition LI1, one can ask whether it is indeed possible to have Lorentz-invariant elements of reality.

To answer this question, let us first note that condition ER1 involves the word 'predict' in an essential way. Since 'predict' refers to the future, i.e. to times later than some instant $t$ in a given Lorentz frame, ER1 is clearly not a relativistically invariant criterion. To get an invariant criterion, we should introduce an invariant specification, i.e. we should make use of the light cone. I have shown in Ref. [9] that the backward light cone is not an appropriate choice, because it does not capture the kind of elements of reality that EPR had in mind. But the forward light cone is. So here's what a relativistically 
invariant sufficient condition for the existence of an element of reality may look like:

If from the (relevant) information on or outside the forward light cone of a possible measurement event $\mathcal{E}$, we can infer with certainty, or at any rate with probability one, the result of measuring a physical quantity at $\mathcal{E}$, then at that event there exists an element of reality corresponding to the physical quantity and having a value equal to the predicted measurement result. [ER3]

Criterion ER3 bears some relation to the Hellwig-Kraus approach [10] to state vector collapse, of which I'll say more in the next section. In that approach, the collapse occurs on the backward light cone of the measurement event. It therefore allows for relevant information obtained through collapse to bear upon a measurement performed in its relative (though not absolute) past, just like ER3 does.

To apply to Hardy's argument, criterion ER3 should also be adapted to nonlocal observables like $U^{+} U^{-}$. How can this be done? Fig. 2 shows two light cones associated with $U^{+}$and $U^{-}$respectively, or more precisely with elements of reality pertaining to these observables at the events in dashed boxes. We can combine the exteriors of the two light cones through their union $\mathcal{U}$ (involving regions 1, 3 and 4) or their intersection $\mathcal{I}$ (involving only region 4$)$.

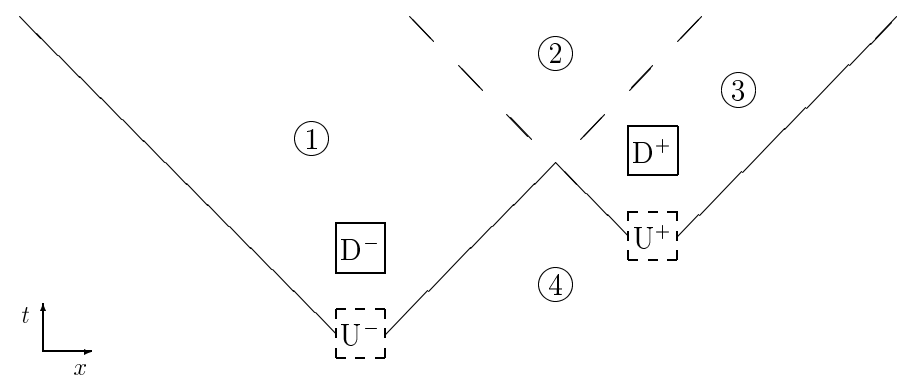

Figure 2: Combination of two light cones

Suppose that we try to adapt criterion ER3 to $\mathcal{U}$. It is clear that any time an actual measurement of $U^{+} U^{-}$is performed, its result can be deduced from information available in $\mathcal{U}$, for this region includes part of the absolute 
future of the measurement events. Hence the criterion is in this case trivial. (We'll turn later to the case where the measurement is not performed.)

Suppose next that we try to adapt criterion ER3 to $\mathcal{I}$. Consider as we did before a run where detection occurs at $D^{+}$and $D^{-}$. Since $D^{-}$is outside the forward light cone of $U^{+}$, ER3 implies that $f\left(U^{+}\right)=1$. Likewise since $D^{+}$is outside the forward light cone of $U^{-}$, ER3 implies that $f\left(U^{-}\right)=1$. This holds in all Lorentz frames.

It turns out, however, that neither $D^{+}$nor $D^{-}$are in $\mathcal{I}$, the intersection of the regions outside the two forward light cones. Hence ER3 cannot be used to deduce the existence of an element of reality associated with $U^{+} U^{-}$, nor a fortiori to attribute a value to $f\left(U^{+} U^{-}\right)$. Hardy's argument therefore no longer goes through, and the product rule (9) no longer necessarily holds.

We should note that criterion ER3 for the existence of elements of reality, and its generalization to nonlocal observables through the region $\mathcal{I}$, involve context [5, 11]. Indeed the 'relevant information' is not the same for the local observable $U^{+}$as it is for the nonlocal observable $U^{+} U^{-}$.

Vaidman [7, 8] has analyzed Hardy's setup using the Aharonov, Bergmann and Lebowitz rule [12], which evaluates the probability of measurement results conditional on postselection as well as preselection. He then found that there is unit probability for the following three intermediate (unperformed) results: (i) $U^{+}$yields 1 ; (ii) $U^{-}$yields 1 ; and (iii) $U^{+} U^{-}$yields 0 . In this sense the product rule for elements of reality does not hold.

In Vaidman's analysis, the value of the element of reality corresponding to $U^{+} U^{-}$can be deduced from information in the union $\mathcal{U}$ of the regions outside the two forward light cones of the events. As he claims, it thus provides Lorentz-invariant elements of reality.

\section{Understanding Hardy's experiment}

Hardy's thought experiment, like many others in quantum mechanics, shows paradoxical features related to correlations over spacelike separations. I have argued elsewhere [13], following others [14], that interpreting quantum mechanics means answering the question, How can the world be for the theory to be true? To attenuate, if not resolve, the paradoxical features of Hardy's setup, let us try to understand it in four different interpretations. 


\subsection{Collapse theories}

Hardy originally framed his argument against Lorentz-invariant elements of reality in the language of state vector collapse. The argument, however, does not depend on that assumption. Nevertheless, collapse theories do provide a way to understand what happens in the experiment.

Assume as before that detectors $D^{+}$and $D^{-}$eventually fire, at instants we shall denote by $t_{+}$and $t_{-}$. For simplicity, we also assume here that detectors $D^{ \pm}$and $C^{ \pm}$are very close to the second beam splitters, so that the time interval between splitting and detection is negligible.

In a nonrelativistic collapse theory such as von Neumann's [15], collapse occurs on an equal time hypersurface. Clearly, this singles out a preferred reference frame, since equal time in one Lorentz frame is not equal time in others.

Let us now consider two different cases. In the first one, the preferred frame is the one where the two detections are simultaneous. Then for $t<$ $t_{+}=t_{-}$, the state vector $|\psi\rangle$ is given by Eq. (6) , whereas for $t>t_{+}=t_{-}$, it is given by $\left|d^{+}\right\rangle\left|d^{-}\right\rangle$. In the second case, the preferred frame is one where detection at $D^{+}$occurs before detection at $D^{-}$, i.e. $t_{+}<t_{-}$. Then we have

$$
|\psi\rangle= \begin{cases}\text { Eq. (6) } & \text { if } t<t_{+}, \\ \left|d^{+}\right\rangle\left|u^{-}\right\rangle & \text {if } t_{+}<t<t_{-} \\ \left|d^{+}\right\rangle\left|d^{-}\right\rangle & \text {if } t_{-}<t\end{cases}
$$

We can see that the observable $U^{-}$is an element of reality in the second case, but not in the first one. This just illustrates the lack of Lorentz invariance of nonrelativistic collapse.

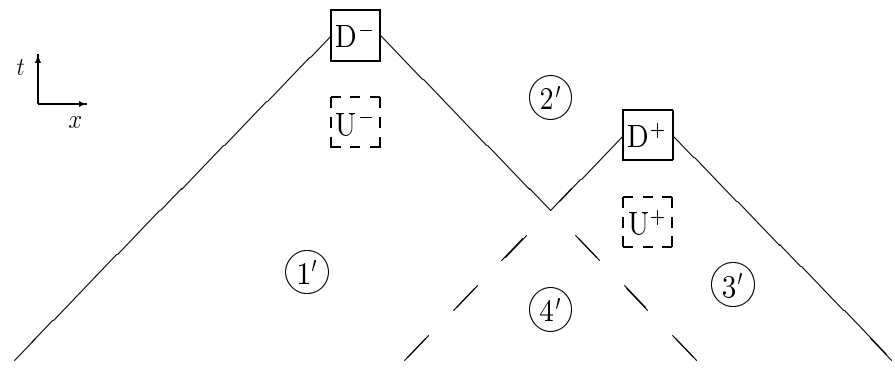

Figure 3: Collapse in the Hellwig-Kraus theory 
The relativistic collapse theory of Hellwig and Krauss [10], applied to the present situation, is illustrated in Fig. 3. In each of the four regions, the state vector is given as follows:

$$
|\psi\rangle= \begin{cases}\left|d^{+}\right\rangle\left|u^{-}\right\rangle & \text {in } 1^{\prime}, \\ \left|d^{+}\right\rangle\left|d^{-}\right\rangle & \text {in } 2^{\prime}, \\ \left|u^{+}\right\rangle\left|d^{-}\right\rangle & \text {in } 3^{\prime}, \\ \text { Eq. (6) } & \text { in } 4^{\prime} .\end{cases}
$$

One can see that $U^{-}$is an element of reality in region $1^{\prime}, U^{+}$is an element of reality in $3^{\prime}$ and $U^{+} U^{-}$is an element of reality in $4^{\prime}$. These regions being different, the product rule doesn't hold.

\subsection{Bohmian mechanics}

In Bohmian mechanics, positions of particles are elements of reality. The electron and the positron both follow deterministic trajectories in one arm of their corresponding interferometers.

The trajectories, however, are not relativistically covariant. There is a preferred frame where they are to be computed. If the preferred frame is the one where both measurements are simultaneous, and detectors $D^{+}$and $D^{-}$ fire, the trajectories can consistently avoid the $u^{+} u^{-}$path. If, on the other hand, the preferred frame is the one where the positron is measured first, then the state vector before the positron measurement is given by Eq. (66). After measurement, it effectively becomes $\left|d^{+}\right\rangle\left|u^{-}\right\rangle$. The electron has gone through $u^{-}$, but one cannot say (as would presumably be found upon explicit calculation) that the positron has gone through $u^{+}$.

Bohmian elements of reality are Lorentz invariant, but condition ER1 is not in general valid. That is, prediction with certainty in one frame is not enough to ascertain the existence of an element of reality.

Note that in Bohmian mechanics, although particle trajectories are not relativistically covariant, statistical predictions are, since they coincide with the ones made by standard quantum mechanics.

\subsection{Everett's relative states}

In Everett's relative-states (or many-worlds) theory [16], the state vector never collapses. All components of the final state vector (7) coexist. Different 
variants of Everett's approach will take the coexistence to apply to different worlds, different minds or different decohering sectors of the state vector.

In a world (say) associated with the $\left|d^{+}\right\rangle\left|d^{-}\right\rangle$component of state vector (7), detectors $D^{+}$and $D^{-}$fire. Elements of reality can be associated with $\left|d^{+}\right\rangle\left\langle d^{+}\right|$and $\left|d^{-}\right\rangle\left\langle d^{-}\right|$only in such worlds. In other worlds just as real as these, however, detectors $D^{+}$and $C^{-}$fire. Elements of reality can there be associated with $\left|d^{+}\right\rangle\left\langle d^{+}\right|$and $\left|c^{-}\right\rangle\left\langle c^{-}\right|$.

In Everett's theory, ER1 is not a sufficient condition for the existence of an element of reality. If $D^{-}$fires in frame $F^{-}$, state vector (4) cannot in general be used to attribute an element of reality to $\left|u^{+}\right\rangle\left\langle u^{+}\right|$. To do so, one would need to put detectors in paths $v^{+}$and $u^{+}$, in addition to detectors $D^{-}$ and $C^{-}$. In that case the various terms of (44) would correspond to different worlds. In all worlds where $D^{-}$would fire, the detector in $u^{+}$would too.

Everett's theory is relativistically covariant. In any reference frame, the positron observer and the electron observer, if they eventually come close along a timelike path, will always find themselves with the proper correlations.

\subsection{Cramer's transactional view}

In Cramer's transactional interpretation [17], the electron-positron source emits a (retarded) offer wave while various detectors (including the gammaray detectors registering electron-positron annihilation) respond with (advanced) confirmation waves. This is reminiscent of Wheeler-Feynman electrodynamics.

Eventually a transaction is established between the source and one detector for each particle. The transaction involves the actual (as opposed to counterfactual) configuration of the measuring devices. Counterfactual reasoning cannot lead to an inference of elements of reality.

The process is relativistically covariant. No element of reality is attached to a trajectory or partial state vector independently of a transaction.

\section{Conclusion}

In quantum mechanics, elements of reality are not easy to reconcile with Lorentz invariance. We have seen that they can if the product rule is abandoned. Different interpretations of quantum mechanics may or may not as- 
sign elements of reality, and if they do they may do so differently. It is the present author's contention, however, that the existence of various possible ways to do so illuminates our understanding of quantum mechanics.

\section{Acknowledgements}

I am thankful to an anonymous referee for comments helping to make the argument clearer. This work was supported by the Natural Sciences and Engineering Research Council of Canada.

\section{References}

[1] A. Einstein, B. Podolsky, N. Rosen, Phys. Rev. 47 (1935) 777.

[2] M. Redhead, Incompleteness, Nonlocality, and Realism (Clarendon Press, Oxford, 1987).

[3] D. Bohm, Phys. Rev. 85 (1952) 166, 180.

[4] L. Hardy, Phys. Rev. Lett. 68 (1992) 2981.

[5] R. Clifton, P. Niemann, Phys. Lett. A 166 (1992) 177.

[6] R. Clifton, C. Pagonis, I. Pitowsky, in: Proc. of the Biennial Meeting of the PSA, Vol. 1 (Philosophy of Science Association, 1992), pp. 114-128.

[7] L. Vaidman, Phys. Rev. Lett. 70 (1993) 3369.

[8] L. Vaidman, arXiv: quant-ph/9703018.

[9] L. Marchildon, Found. Phys. 38 (2008) 804.

[10] K.E. Hellwig, K. Kraus, Phys. Rev. D 1 (1970) 566.

[11] K. Berndl, S. Goldstein, Phys. Rev. Lett. 72 (1994) 780.

[12] Y. Aharonov, P.G. Bergmann, J.L. Lebowitz, Phys. Rev. 134 (1964) B1410.

[13] L. Marchildon, Found. Phys. 34 (2004) 1453. 
[14] B.C. van Fraassen, Quantum Mechanics: An Empiricist View (Clarendon Press, Oxford, 1991).

[15] J. von Neumann, Mathematical Foundations of Quantum Mechanics (Princeton University Press, Princeton, 1955).

[16] H. Everett III, Rev. Mod. Phys. 29 (1957) 454.

[17] J.G. Cramer, Rev. Mod. Phys. 58 (1986) 647. 